On the Superlinear Convergence of Interior Point Algorithms for a General Class of Problems

\author{
Y. Zhang \\ R.A. Tapia \\ F. Potra
}

March, 1990

(Revised October 1991)

TR90-9 



\title{
On the Superlinear Convergence of Interior-Point Algorithms for a General Class of Problems*
}

\author{
Yin Zhang $\stackrel{\dagger}{\dagger}$ Richard Tapia ${ }^{\ddagger}$ and Florian Potra ${ }^{\S}$ \\ March 1990 (Revised October, 1991)
}

\begin{abstract}
In this paper, we extend the $Q$-superlinear convergence theory recently developed by Zhang, Tapia and Dennis for a class of interior-point linear programming algorithms to similar interior-point algorithms for quadratic programming and for linear complementarity problems. Our unified approach consists of viewing all these algorithms as a damped Newton method applied to perturbations of a general problem. We establish a set of sufficient conditions for these algorithms to achieve $Q$-superlinear convergence. The key ingredients consist of asymptotically taking the step to the boundary of the positive orthant and letting the centering parameter approach zero at a specific rate. The construction of algorithms that have both the global property of polynomiality and the local property of superlinear convergence will be the subject of further research.
\end{abstract}

Keywords: interior-point algorithms, Linear programming, Quadratic programming, Linear complementarity problems, $Q$-superlinear convergence.

Abbreviated Title: Superlinear Convergence for Interior-point Algorithms

*Research supported in part by NSF Coop. Agr. No. CCR-8809615, AFOSR 89-0363, DOE DEFG0586ER25017 and ARO 9DAAL03-90-G-0093.

${ }^{\dagger}$ Department of Mathematics and Statistics, University of Maryland Baltimore County, Baltimore, MD 21228

$\ddagger$ Department of Mathematical Sciences, Rice University, Houston, Texas, 77251-1892

$\S$ Department of Mathematics, The University of Iowa, Iowa City, Iowa 52242 



\section{Introduction}

Consider the general nonlinear system

$$
F(x, y)=\left(\begin{array}{c}
M x+N y-h \\
X Y e
\end{array}\right)=0, \quad(x, y) \geq 0
$$

where $x, y, h, e \in \mathbf{R}^{n}, M, N \in \mathbf{R}^{n \times n}, X=\operatorname{diag}(x), Y=\operatorname{diag}(y)$ and $e$ has all components equal to one.

We call the following set the feasibility set of problem (1.1):

$$
\Omega=\left\{(x, y): x, y \in \mathbf{R}^{n}, M x+N y=h,(x, y) \geq 0\right\}
$$

A feasible pair $(x, y) \in \Omega$ is said to be strictly feasible if it is positive. In this work we tacitly assume that the relative interior of $\Omega$ is nonempty, i.e., strictly feasible points exist.

Problem (1.1) is sufficiently general to include linear and quadratic programming problems, and linear complementarity problems. Observe that if $N=-I$, then this problem is the standard linear complementarity problem (LCP). Moreover, the assumption that $M$ is positive semi-definite will be sufficient to guarantee that the algorithms under investigation produce well-defined iterates (Corollary 2.1).

It is well-known that quadratic programs are special cases of linear complementarity problems. We now provide a somewhat different formulation of quadratic programs as special cases of Problem (1.1) instead of those of the standard LCP. Consider the quadratic program (QP)

$$
\begin{array}{cl}
\operatorname{minimize} & c^{T} x+\frac{1}{2} x^{T} Q x \\
\text { subject to } & A x=b \\
& x \geq 0
\end{array}
$$

where $c, x \in \mathbf{R}^{n}, b \in \mathbf{R}^{m}, A \in \mathbf{R}^{m \times n}(m<n)$ and has full row rank, and $Q \in \mathbf{R}^{n \times n}$ is symmetric. In Corollary 2.1, we will demonstrate that iterates produced by the algorithms under investigation are well-defined if $Q$ is positive semi-definite on the null space of $A$. In this case, it is well-known that the problem is convex and the first order conditions are both necessary and sufficient for optimality. The first-order conditions for (1.2) can be transformed into the form of (1.1). To see this, let $B \in \mathbf{R}^{(n-m) \times n}$ be any matrix such that the columns of 
$B^{T}$ form a basis for the null space of $A$. The first-order conditions for the quadratic program (1.2) are (see Dantzig [1])

$$
\left(\begin{array}{c}
A x-b \\
A^{T} \lambda-Q x+y-c \\
X Y e
\end{array}\right)=0, \quad(x, y) \geq 0,
$$

where $\lambda$ and $y$ are the dual variables. To eliminate the dual variables $\lambda$ from the above system, we pre-multiply the second equation by the nonsingular matrix $\left[A^{T} B^{T}\right]^{T}$. Noticing that $B A^{T}=0$, we obtain

$$
0=\left[\begin{array}{l}
A \\
B
\end{array}\right]\left(A^{T} \lambda-Q x+y-c\right)=\left(\begin{array}{c}
A A^{T} \lambda-A(Q x-y+c) \\
-B Q x+B y-B c
\end{array}\right) .
$$

Since $A A^{T}$ is nonsingular, $\lambda$ is uniquely determined once $x$ and $y$ are known. Removing the equation for $\lambda$, we arrive at the following $2 n$-dimensional nonlinear system with nonnegativity constraints for $(x, y)$

$$
\left(\begin{array}{c}
A x-b \\
-B Q x+B y-B c \\
X Y e
\end{array}\right)=0, \quad(x, y) \geq 0 .
$$

Clearly, (1.4) is in the form of (1.1) with

$$
M=\left[\begin{array}{c}
A \\
-B Q
\end{array}\right], N=\left[\begin{array}{c}
0 \\
B
\end{array}\right] \text { and } h=\left[\begin{array}{c}
b \\
B c
\end{array}\right]
$$

When $Q=0$, the quadratic program (1.2) reduces to a standard-form linear program (LP)

$$
\begin{array}{ll}
\operatorname{minimize} & c^{T} x \\
\text { subject to } & A x=b \\
& x \geq 0 .
\end{array}
$$

Hence (1.2) also includes the linear program. However, because of the importance of linear programming in optimization, we will state results for linear programming separately; fully aware that they are special cases of quadratic programming. We have shown that the framework of problem (1.1) is quite general. 
The objective of this work is to analyze the asymptotic behavior of a generic interiorpoint algorithm for solving problem (1.1). More specifically, we will study the $Q$-convergence rate of this general algorithm. The issues of global convergence and complexity are not of concern here.

Recently, Zhang, Tapia and Dennis [18, see Theorem 3.1] established a $Q$-superlinear convergence theory for a class of primal-dual interior-point algorithm for linear programming. In this paper, we extend their result to the general problem (1.1) and therefore extend the result to quadratic programming and linear complementarity problems. In spite of its close connection to [18], we have made this paper self-contained.

Given $u, v \in \mathbf{R}^{n}$ and $\eta \in \mathbf{R}$, We will use the notation:

$$
\min (u)=\min _{1 \leq i \leq n}[u]_{i} \text { and } \min (u, v, \eta)=\min \{\min (u), \min (v), \eta\},
$$

where $[u]_{i}$ denotes the $i$-th component of $u$.

The paper is organized as follows. In Section 2, we describe a general interior-point algorithmic framework for (1.1). Then in Section 3, we present our superlinear convergence rate result. Concluding remarks are given in Section 4.

\section{Algorithm}

It is now fairly well understood how a class of interior-point algorithms can be viewed as damped Newton methods and that the inclusion of the logarithmic barrier term (so-called centering) can be viewed as perturbing the right-hand side of the Newton system. Indeed, Zhang, Tapia and Dennis [18] focused on issues concerning how fast the damped Newton method could approach the Newton method (i.e., step-length approaches one), and how fast the perturbation term (barrier parameter) should be phased out so that the fast convergence of Newton's method is not compromised. Their work covered linear programming applications. As previously mentioned, the objective of the present work is to extend a particular nice part of their superlinear convergence theory to quadratic programming and linear complementarity problems. Our vehicle for accomplishing this objective is the use of the general problem (1.1). We assume that the reader is familiar with the above algorithmic 
considerations and therefore present our algorithmic framework with no further motivation or explanation.

Recall that $F(x, y)$ is given by (1.1).

Algorithm 1 Given a pair $\left(x_{0}, y_{0}\right)>0$. For $k=0,1,2, \ldots$, do

(1) Choose $\sigma_{k} \in[0,1)$ and $\tau_{k} \in(0,1)$. Set $\mu_{k}=\sigma_{k} x_{k}^{T} y_{k} / n$.

(2) Solve the following system for $\left(\Delta x_{k}, \Delta y_{k}\right)$ :

$$
F^{\prime}\left(x_{k}, y_{k}\right)\left(\begin{array}{c}
\Delta x \\
\Delta y
\end{array}\right)=-F\left(x_{k}, y_{k}\right)+\left(\begin{array}{c}
0 \\
\mu_{k} e
\end{array}\right) \text {. }
$$

(3) Compute the step-length:

$$
\alpha_{k}=\frac{-\tau_{k}}{\min \left(X_{k}^{-1} \Delta x_{k}, Y_{k}^{-1} \Delta y_{k},-\tau_{k}\right)}
$$

(4) Update: $x_{k+1}=x_{k}+\alpha_{k} \Delta x_{k}$ and $y_{k+1}=y_{k}+\alpha_{k} \Delta y_{k}$.

Notice that in Algorithm 1, we do not require that the starting point $\left(x_{0}, y_{0}\right)$ be feasible. Also notice that without the perturbation term $\mu_{k} e$ in the right-hand side of $(2.1)$, the search direction $\left(\Delta x_{k}, \Delta y_{k}\right)$ is the Newton step. We always have $0<\alpha_{k} \leq 1$. Moreover, $\alpha_{k}=1$ if and only if $\min \left(X_{k}^{-1} \Delta x_{k}, Y_{k}^{-1} \Delta y_{k}\right) \geq-\tau_{k}$. We should expect that only in rare cases would the full Newton step lead to a strictly positive iterate; hence we should expect in most cases to have $\alpha_{k}<1$ where $\alpha_{k}$ is given by (2.2). The choice $\tau_{k}=1$ corresponds to allowing steps to the boundary of the positive orthant and a loss of strict feasibility. Therefore, it is natural to view Algorithm 1 as a perturbed and damped Newton's method. We see that if $\left(x_{0}, y_{0}\right)$ is in $\Omega$, then the iteration sequence $\left\{\left(x_{k}, y_{k}\right)\right\}$ will be strictly feasible. In the case of linear programming, there are no linear equations in $F(x, y)$ that involve both $x$ and $y$. If $\left(x_{k}, y_{k}\right) \in \Omega$, then different step-lengths can be used to update $x_{k}$ and $y_{k}$ and still retain strictly feasible $\left(x_{k+1}, y_{k+1}\right)$. This strategy has been shown to be more efficient in practice (see Lustig, Marsten and Shanno [9], for example). However, it will not affect our results since our analysis will show that as long as $\tau_{k} \rightarrow 1$ both step-lengths will converge to one. 
Algorithm 1 covers or is closely related to a wide range of existing interior-point algorithms for linear programming, quadratic programming and linear complementarity problems. In particular, it covers most of the existing primal-dual interior-point algorithms for linear programming as well as quadratic programming, including Kojima, Mizuno and Yoshise [7], Todd and Ye [15], Monteiro and Adler [12, 13], Lustig [8], Gonzaga and Todd [2], Mizuno, Todd and Ye [11]. Algorithms for linear complementarity problems that are covered by or closely related to Algorithm 1 include Kojima, Mizuno and Yoshise [5, 6], Kojima, Megiddo and Noma [3], and Kojima, Mizuno and Noma [4].

Although these algorithms have been motivated and presented in various ways including path-following (homotopy or continuation), potential reduction or affine scaling algorithms, most of them fit into the framework of the perturbed and damped Newton's method applied to the general problem (1.1). Due to the extensive activity in this area, our list of references is not complete. For a more complete list of references, especially in the cases of quadratic programming and linear complementarity problems, we refer the reader to two recent survey papers by Ye $[16,17]$.

The following proposition gives a condition which guarantees that the iterates produced by Algorithm 1 are well-defined.

Proposition 2.1 The iterates produced by Algorithm 1 are well-defined if for any positive diagonal matrix $D \in \mathbf{R}^{n \times n}$, the matrix $N-M D$ is nonsingular.

Proof: Since $\left(x_{0}, y_{0}\right)>0$ and

$$
F^{\prime}(x, y)=\left[\begin{array}{ll}
M & N \\
Y & X
\end{array}\right]
$$

the nonsingularity of $F^{\prime}\left(x_{0}, y_{0}\right)$ is equivalent to that of

$$
\left[\begin{array}{cc}
I & -M Y_{0}^{-1} \\
0 & I
\end{array}\right] F^{\prime}\left(x_{0}, y_{0}\right)=\left[\begin{array}{cc}
0 & N-M Y_{0}^{-1} X_{0} \\
Y_{0} & X_{0}
\end{array}\right]
$$

This latter matrix is nonsingular if and only if $N-M Y_{0}^{-1} X_{0}$ is nonsingular. By our condition, $\left(x_{1}, y_{1}\right)$ is well-defined. An induction argument completes the proof.

The following corollary is well-known and one can easily verify that Proposition 2.1 is satisfied in the three cases of interest. 
Corollary 2.1 The iterates produced by Algorithm 1 are well-defined for

1. the linear complementarity problem $(N=-I)$ with $M$ positive semi-definite,

2. the quadratic programming problem (1.2) with $Q$ positive semi-definite on the null space of $A$,

3. the linear programming problem (1.6).

We should mention that we have stated Algorithm 1 in the current form purely for the purposes of obtaining a unified theory and notational convenience. By directly applying the perturbed and damped Newton method to the first order conditions for the quadratic program (1.2), it is not difficult to see that an identical iteration sequence $\left\{\left(x_{k}, y_{k}\right)\right\}$ will be generated without eliminating the dual variable $\lambda$ and introducing the matrix $B$.

\section{Superlinear Convergence}

The literature contains numerous studies directed at investigating the convergence properties of interior-point algorithms covered by or closely related to Algorithm 1. However, most of these studies were concerned only with the issues of global convergence and complexity. The issue of convergence rate, which is certainly important, has not been thoroughly studied for many interior-point algorithms. One of the few papers that studied asymptotic behavior (local convergence) of interior-point algorithms is Kojima, Megiddo and Noma [3]. In their paper, Kojima, Megiddo and Noma proved that for a class of complementarity problems, $Q$-linear, in addition to global convergence, superlinear and quadratic local convergence can be achieved by some interior-point algorithms in the form of Algorithm 1. However, all their convergence rate results were obtained under the restriction that the Jacobian matrix $F^{\prime}(x, y)$ was nonsingular at the solution. In this section, we provide a set of sufficient conditions for superlinear convergence of Algorithm 1 applied to the general problem (1.1). These conditions do not require the nonsingularity of $F^{\prime}(x, y)$ at solutions. How to apply these conditions to construct globally and superlinearly convergent algorithms is an interesting topic and the subject of further research. 
It is satisfying that it is possible to obtain a superlinear convergence rate without the assumption of nonsingularity of the Jacobian matrix at the solution. In the case of linear programming, this allows one to avoid restrictive nondegeneracy assumptions. The motivation for this theory came from numerical experiments that demonstrated superlinear convergence even for highly degenerate linear programs.

At the $k$-th iteration of Algorithm 1, let

$$
\eta_{k}=\frac{x_{k}^{T} y_{k} / n}{\min \left(X_{k} Y_{k} e\right)}
$$

Since $x_{k}^{T} y_{k} / n$ is the average value of the elements of $X_{k} Y_{k} e$, it is clear that $\eta_{k} \geq 1$.

Theorem 3.1 Let $\left\{\left(x_{k}, y_{k}\right)\right\}$ be generated by Algorithm 1 with $\tau_{k} \rightarrow 1$ and $\sigma_{k} \rightarrow 0$, and let $\left(x_{k}, y_{k}\right) \rightarrow\left(x_{*}, y_{*}\right)$. Assume

(i) strict complementarity,

(ii) the sequence $\left\{\eta_{k}\right\}$ is bounded,

(iii) there exists $\rho \in[0,1)$ such that for $k$ sufficiently large

$$
\Delta x_{k}^{T} \Delta y_{k} \geq-\frac{\rho}{2}\left(\Delta x_{k}^{T}\left(X_{k}^{-1} Y_{k}\right) \Delta x_{k}+\Delta y_{k}^{T}\left(X_{k} Y_{k}^{-1}\right) \Delta y_{k}\right)
$$

Then $\left(x_{*}, y_{*}\right)$ solves problem (1.1) and the sequence $\left\{F\left(x_{k}, y_{k}\right)\right\}$ component-wise converges to zero $Q$-superlinearly. Furthermore, the sequence $\left\{F\left(x_{k}, y_{k}\right)\right\}$ is $Q$-superlinearly convergent, i.e., for any norm

$$
\lim _{k \rightarrow \infty} \sup \frac{\left\|F\left(x_{k+1}, y_{k+1}\right)\right\|}{\left\|F\left(x_{k}, y_{k}\right)\right\|}=0
$$

Before we prove Theorem 3.1, we would like to comment on the assumptions of Theorem 3.1. First, Assumption (iii) is not particularly restrictive since we will see later that in the context of linear programming, quadratic programming with $Q$ positive semi-definite on the null space of $A$ and linear complementarity problems with $M$ positive semi-definite, we have the stronger result that $\Delta x_{k}^{T} \Delta y_{k} \geq 0$ for $\left(x_{k}, y_{k}\right) \in \Omega$. We used the more general Assumption (iii) instead of $\Delta x_{k}^{T} \Delta y_{k} \geq 0$ based on the consideration that the former could be useful in studying situations where $\left(x_{k}, y_{k}\right)$ is not feasible. We stress that the algorithm 
designer is free to choose $\sigma_{k}$ and $\tau_{k}$, and the requirement that they be chosen so that $\sigma_{k} \rightarrow 0$ and $\tau_{k} \rightarrow 1$ is not particular restrictive.

On the other hand, the compatibility of Assumptions (ii) with the choices $\tau_{k} \rightarrow 1$ and $\sigma_{k} \rightarrow 0$ may be a cause for concern. It seems as if letting $\tau_{k} \rightarrow 1$ and $\sigma_{k} \rightarrow 0$ might force $\eta_{k} \rightarrow \infty$. However, our numerical experience has shown this not to be the case for linear programming. In our numerical studies with Netlib problems for linear programming, we let $\tau_{k} \rightarrow 1$ and $\sigma_{k} \rightarrow 0$ and always observed strict complementarity and bounded $\left\{\eta_{k}\right\}$. While on occasion we saw some rather large values for $\eta_{k}$ 's, they eventually leveled off or actually started to decrease as the iterates approached a solution. We did not observe continued growth in the values of $\eta_{k}$ as our algorithm converged. Moreover, the observed convergence was clearly $Q$-superlinear and $\alpha_{k} \rightarrow 1$. Of course, the behavior of $\left\{\eta_{k}\right\}$ varies with several factors including how fast $\left\{\tau_{k}\right\}$ converges to one and $\left\{\sigma_{k}\right\}$ to zero. We do not mean to imply that unbounded $\left\{\eta_{k}\right\}$ cannot occur. Instead, we feel that it appears to be more the exception than the rule in linear programming. It still remains to be seen whether or not this same phenomenon exists in quadratic programming and linear complementarity problems. There is no doubt that this topic merits further study.

To prove Theorem 3.1 , we need the following lemma.

Lemma 3.1 Under the assumptions of Theorem 3.1,

$$
\lim _{k \rightarrow \infty} \alpha_{k}=1 \text {. }
$$

Proof: Define at each iteration

$$
p_{k}=X_{k}^{-1} \Delta x_{k}, \text { and } q_{k}=Y_{k}^{-1} \Delta y_{k} .
$$

At iteration $k$, from (2.1) and (2.3) we have

$$
Y_{k} \Delta x_{k}+X_{k} \Delta y_{k}=-X_{k} Y_{k} e+\mu_{k} e,
$$

or equivalently, recalling that $\mu_{k}=\sigma_{k} x_{k}^{T} y_{k} / n$ (see Step (1) of Algorithm 1)

$$
p_{k}+q_{k}=-e+\mu_{k}\left(X_{k} Y_{k}\right)^{-1} e=-e+\sigma_{k} T_{k} e,
$$

where $T_{k}=\left(x_{k}^{T} y_{k} / n\right)\left(X_{k} Y_{k}\right)^{-1}$. Since $\eta_{k}=\left\|T_{k} e\right\|_{\infty}$, Assumptions (ii) and $\sigma_{k} \rightarrow 0$ imply

$$
\lim _{k \rightarrow \infty}\left(p_{k}+q_{k}\right)=-e \text {. }
$$


Multiply both sides of $(3.3)$ by $\left(X_{k} Y_{k}\right)^{-\frac{1}{2}}$ and consider the square of the $\ell_{2}$-norm. We have the following equality

$$
\left\|\left(X_{k} Y_{k}\right)^{\frac{1}{2}} p_{k}\right\|_{2}^{2}+\left\|\left(X_{k} Y_{k}\right)^{\frac{1}{2}} q_{k}\right\|_{2}^{2}+2 \Delta x_{k}^{T} \Delta y_{k}=x_{k}^{T} y_{k}\left(1-2 \sigma_{k}+\sigma_{k}^{2} \frac{x_{k}^{T} y_{k}}{n} \frac{e^{T}\left(X_{k} Y_{k}\right)^{-1} e}{n}\right)
$$

Note that

$$
\left\|\left(X_{k} Y_{k}\right)^{\frac{1}{2}} p_{k}\right\|_{2}^{2}=\Delta x_{k}^{T}\left(X_{k}^{-1} Y_{k}\right) \Delta x_{k} \text { and }\left\|\left(X_{k} Y_{k}\right)^{\frac{1}{2}} q_{k}\right\|_{2}^{2}=\Delta y_{k}^{T}\left(X_{k} Y_{k}^{-1}\right) \Delta y_{k}
$$

By Assumption (iii),

$$
(1-\rho)\left(\left\|\left(X_{k} Y_{k}\right)^{\frac{1}{2}} p_{k}\right\|_{2}^{2}+\left\|\left(X_{k} Y_{k}\right)^{\frac{1}{2}} q_{k}\right\|_{2}^{2}\right) \leq x_{k}^{T} y_{k}\left(1-2 \sigma_{k}+\sigma_{k}^{2} \frac{x_{k}^{T} y_{k}}{n} \frac{e^{T}\left(X_{k} Y_{k}\right)^{-1} e}{n}\right) .
$$

Dividing the above inequality by $x_{k}^{T} y_{k} / n$, we obtain

$$
(1-\rho)\left(\left\|T_{k}^{-\frac{1}{2}} p_{k}\right\|_{2}^{2}+\left\|T_{k}^{-\frac{1}{2}} q_{k}\right\|_{2}^{2}\right) \leq n\left(1-2 \sigma_{k}+\sigma_{k}^{2} \frac{e^{T} T_{k} e}{n}\right) .
$$

Assumption (ii) implies that $\left\{\left\|T_{k}\right\|\right\}$ is bounded above and $\left\{\left\|T_{k}^{-\frac{1}{2}}\right\|\right\}$ is bounded away from zero. Therefore, from (3.5) both $\left\{p_{k}\right\}$ and $\left\{q_{k}\right\}$ are bounded. It now follows from (2.2) that $\left\{\alpha_{k}\right\}$ is bounded away from zero.

Now assume $\left[x_{*}\right]_{i}>0$. Obviously,

$$
1=\lim _{k \rightarrow \infty} \frac{\left[x_{k+1}\right]_{i}}{\left[x_{k}\right]_{i}}=\lim _{k \rightarrow \infty}\left(1+\alpha_{k}\left[p_{k}\right]_{i}\right)
$$

This implies $\left[p_{k}\right]_{i} \rightarrow 0$, because $\left\{\alpha_{k}\right\}$ is bounded away from zero. From (3.4) we have $\left[q_{k}\right]_{i} \rightarrow-1$. On the other hand, if $\left[x_{*}\right]_{i}=0$, then $\left[y_{*}\right]_{i}>0$ by strict complementarity. The same argument, interchanging the roles of $p_{k}$ and $q_{k}$, gives $\left[q_{k}\right]_{i} \rightarrow 0$ and $\left[p_{k}\right]_{i} \rightarrow-1$. Therefore, the components of $p_{k}$ and $q_{k}$ converge to either 0 or -1 . Consequently, from (3.2), (2.2) and $\tau_{k} \rightarrow 1$ it follows that $\alpha_{k} \rightarrow 1$. This completes the proof.

Now we are ready to prove Theorem 3.1 .

Proof of Theorem 3.1: Let

$$
F_{1}(x, y)=M x+N y-h \text { and } F_{2}(x, y)=X Y e
$$

We will prove that both $\left\{F_{1}\left(x_{k}, y_{k}\right)\right\}$ and $\left\{F_{2}\left(x_{k}, y_{k}\right)\right\}$ component-wise converge to zero $Q$-superlinearly. This will imply that $\left\{F\left(x_{k}, y_{k}\right)\right\}$ component-wise converges to zero $Q$ superlinearly. It is not difficult to see that component-wise $Q$-superlinear convergence of a vector sequence implies its $Q$-superlinear convergence. 
First we show that $\left\{F_{1}\left(x_{k}, y_{k}\right)\right\}$ component-wise converges to zero $Q$-superlinearly. If $F_{1}\left(x_{0}, y_{0}\right)=0$ (i.e., $\left(x_{0}, y_{0}\right)$ is a feasible starting point), then it is easy to see that $F_{1}\left(x_{k}, y_{k}\right)=$ 0 for all $k$. Therefore, we need only consider the case where $F_{1}\left(x_{0}, y_{0}\right) \neq 0$. Note that Newton's method solves linear equations in one step. If for some integer $p \geq 0, \alpha_{p}=1$, then we have $F_{1}\left(x_{k}, y_{k}\right)=0$ for all $k>p$. Therefore, we need only consider the case where $\alpha_{k}<1$ for all $k$. It is easy to see from Steps (2) and (4) of Algorithm 1 that

$$
F_{1}\left(x_{k+1}, y_{k+1}\right)=\left(M x_{k}+N y_{k}-h\right)+\alpha_{k}\left(M \Delta x_{k}+N \Delta y_{k}\right)=\left(1-\alpha_{k}\right) F_{1}\left(x_{k}, y_{k}\right)
$$

Since $\alpha_{k} \rightarrow 1,\left\{F_{1}\left(x_{k}, y_{k}\right)\right\}$ component-wise converges to zero $Q$-superlinearly.

Next, we show that $\left\{F_{2}\left(x_{k}, y_{k}\right)\right\}$ component-wise converges to zero $Q$-superlinearly. From Step (4) of Algorithm 1,

$$
X_{k}^{-1} x_{k+1}=e+\alpha_{k} p_{k} \text { and } Y_{k}^{-1} y_{k+1}=e+\alpha_{k} q_{k}
$$

Adding the above two equations, we have

$$
X_{k}^{-1} x_{k+1}+Y_{k}^{-1} y_{k+1}=2 e+\alpha_{k}\left(p_{k}+q_{k}\right) \text {. }
$$

It follows from (3.4) and $\alpha_{k} \rightarrow 1$ that

$$
\lim _{k \rightarrow \infty}\left(X_{k}^{-1} x_{k+1}+Y_{k}^{-1} y_{k+1}\right)=e
$$

If $\left[x_{*}\right]_{i}=0$, then by strict complementarity, $\left[y_{*}\right]_{i}>0$ and $\left[y_{k+1}\right]_{i} /\left[y_{k}\right]_{i} \rightarrow 1$. It follows from (3.6) that $\left[x_{k+1}\right]_{i} /\left[x_{k}\right]_{i} \rightarrow 0$. Therefore, $\left[x_{k}\right]_{i} \rightarrow 0 Q$-superlinearly. By the symmetry of the relation (3.6), we have $\left[y_{k}\right]_{j} \rightarrow 0 Q$-superlinearly if $\left[y_{*}\right]_{j}=0$. Thus, all variables that converge to zero do so $Q$-superlinearly. That is, for each index $i$ either

$$
\lim _{k \rightarrow \infty} \frac{\left[x_{k+1}\right]_{i}}{\left[x_{k}\right]_{i}}=0 \text { and } \lim _{k \rightarrow \infty} \frac{\left[y_{k+1}\right]_{i}}{\left[y_{k}\right]_{i}}=1
$$

or

$$
\lim _{k \rightarrow \infty} \frac{\left[x_{k+1}\right]_{i}}{\left[x_{k}\right]_{i}}=1 \text { and } \lim _{k \rightarrow \infty} \frac{\left[y_{k+1}\right]_{i}}{\left[y_{k}\right]_{i}}=0 .
$$

In either case, for every index $i$,

$$
\lim _{k \rightarrow \infty} \frac{\left[x_{k+1}\right]_{i}\left[y_{k+1}\right]_{i}}{\left[x_{k}\right]_{i}\left[y_{k}\right]_{i}}=\lim _{k \rightarrow \infty} \frac{\left[X_{k+1} Y_{k+1} e\right]_{i}}{\left[X_{k} Y_{k} e\right]_{i}}=0
$$


We have proved that $\left\{\left[X_{k} Y_{k} e\right]_{i}\right\}$ converges to zero $Q$-superlinearly for every index $i$. As was mentioned above, the component-wise $Q$-superlinear convergence of $\left\{F\left(x_{k}, y_{k}\right)\right\}$ implies its $Q$-superlinear convergence. This completes the proof.

A key idea in the proof of Theorem 3.1 can be traced back to a work by Tapia in 1980 [14]. In that paper, Tapia pointed out [14, Theorem 3] that an algorithm which at each iteration satisfies the Taylor linearization of the complementarity equation has the property that the variables that converge to zero do so $Q$-superlinearly. This result assumed strict complementarity and step-length one. Observe that (3.6) is equivalent to

$$
X_{k} Y_{k} e+Y_{k}\left(x_{k+1}-x_{k}\right)+X_{k}\left(y_{k+1}-y_{k}\right) \rightarrow 0
$$

We see that the Taylor linearization of complementarity is satisfied asymptotically in our situation.

The following theorem deals with the $Q$-superlinear convergence of Algorithm 1 applied to linear complementarity problems, quadratic programming and linear programming.

Theorem 3.2 Let $\left\{\left(x_{k}, y_{k}\right)\right\}$ be generated by Algorithm 1 with $\tau_{k} \rightarrow 1$ and $\sigma_{k} \rightarrow 0$, and let $\left(x_{k}, y_{k}\right) \rightarrow\left(x_{*}, y_{*}\right)$. Under Assumptions (i)-(ii) of Theorem 3.1, if $\left(x_{p}, y_{p}\right) \in \Omega$ for some $p$, then $\left(x_{*}, y_{*}\right)$ solves problem (1.1) and the sequence $\left\{F\left(x_{k}, y_{k}\right)\right\}$ component-wise converges to zero $Q$-superlinearly for the following three cases:

1. the linear complementarity problem $(N=-I)$ with $M$ positive semi-definite,

2. the quadratic programming problem (1.2) with $Q$ positive semi-definite on the null space of $A$,

3. the linear programming problem (1.6).

Proof: We need to prove that Assumption (iii) of Theorem 3.1 is satisfied for each of the above three cases. Observe that for all $k \geq p$ we have $\left(x_{k}, y_{k}\right) \in \Omega$ and $M \Delta x_{k}+N \Delta y_{k}=0$ (see (2.1)). It suffices to prove that $u^{T} v \geq 0$ for all $u, v \in \mathbf{R}^{n}$ satisfying $M u+N v=0$.

In the first case $(N=-I), M u+N v=0$ is equivalent to $v=M u$. Hence $u^{T} v=$ $u^{T} M u \geq 0$ because $M$ is positive semi-definite.

In the second case (see (1.5)), $M u+N v=0$ is equivalent to $A u=0$ and $B Q u=B v$. Using the representations $u=B^{T} u_{2}$ and $v=A^{T} v_{1}+B^{T} v_{2}$, where $v_{1} \in \mathbf{R}^{m}$ and $u_{2}, v_{2} \in \mathbf{R}^{n-m}$, 
and noticing that $A^{T} \perp B^{T}$, we have $u^{T} v=u_{2}^{T} B B^{T} v_{2}$. Moreover, $B Q u=B v$ is equivalent to $B Q B^{T} u_{2}=B B^{T} v_{2}$. Hence, if $Q$ is positive semi-definite in the null space of $A$, then

$$
u^{T} v=u_{2}^{T} B B^{T} v_{2}=u_{2}^{T}\left(B Q B^{T}\right) u_{2} \geq 0
$$

The third case follows immediately from the fact that $Q=0$ is positive semi-definite.

It is worth noting that feasibility is assumed in Theorem 3.2 but not in Theorem 3.1. It is not clear if Assumption (iii) of Theorem 3.1 may be satisfied without feasibility. This topic perhaps deserves more study because infeasible starting points are used in most practical implementations.

\section{Concluding Remarks}

The generality of problem (1.1) and the perturbed and damped Newton's method viewpoint have enabled us to analyze the local convergence behavior of a class of interior-point algorithms for linear programming, quadratic programming and linear complementarity problems in a unified approach.

We developed a $Q$-superlinear convergence theory that does not assume any information on the Jacobian matrix at the solution. This theory was used to establish sufficient conditions for $Q$-superlinear convergence of a class of interior-point algorithms for linear programming, quadratic programming (with $Q$ positive semi-definite on the null space of $A$ ) and positive semi-definite linear complementarity problems.

\section{Acknowledgment}

We thank two anonymous referees for their constructive comments.

\section{References}

[1] G. B. Dantzig. Linear Programming and Extensions. Princeton University Press, Princeton, NJ, 1963. 
[2] C. C. Gonzaga and M.J. Todd. An $O(\sqrt{n} L)$-iteration large-step primal-dual affine algorithm for linear programming. Technical Report 862, School of Operations Research and Industrial Engineering, Cornell University, 1989.

[3] M. Kojima, N. Megiddo, and T. Noma. Homotopy continuation methods for complementarity problems, 1988. manuscript, IBM Almaden Research Center, San Jose, California.

[4] M. Kojima, S. Mizuno, and T. Noma. A new continuation method for complementarity problems with uniform $p$-functions. Mathematical Programming, 43:107-113, 1989.

[5] M. Kojima, S. Mizuno, and A. Yoshise. An $O(\sqrt{n} L)$ iteration potential reduction algorithm for linear complementarity problems. Mathematical Programming, 50:331$342,1991$.

[6] M. Kojima, S. Mizuno, and A. Yoshise. A primal-dual algorithm for a class of linear complementarity problems. Mathematical Programming, 44:1-26, 1989.

[7] M. Kojima, S. Mizuno, and A. Yoshise. A primal-dual interior point method for linear programming. In Nimrod Megiddo, editor, Progress in Mathematical programming, interior-point and related methods, pages 29-47. Springer-Verlag, New York, 1989.

[8] I.J. Lustig. A generic primal-dual interior point algorithm. Technical Report SOR 88-3, Dept. Civil Eng. and O.R., Princeton University, 1988.

[9] I.J. Lustig, R.E. Marsten, and D.F. Shanno. Computational experience with a primaldual interior point method for linear programming. Journal of Linear Algebra and Applications, 152:191-222, 1991.

[10] N. Megiddo. Pathways to the optimal set in linear programming. In Nimrod Megiddo, editor, Progress in Mathematical programming, interior-point and related methods, pages 131-158. Springer-Verlag, New York, 1989.

[11] S. Mizuno, M.J. Todd, and Y. Ye. On adaptive step primal-dual interior-point algorithms for linear programming. Technical Report 944, School of Operations Research and Industrial Engineering, Cornell University, 1989. To appear in Math. of O.R.. 
[12] R.C. Monteiro and I. Adler. Interior path-following primal-dual algorithms. Part I: linear programming. Math. Prog., 44:27-41, 1989.

[13] R.C. Monteiro and I. Adler. Interior path-following primal-dual algorithms. Part II: convex quadratic programming. Math. Prog., 44:43-66, 1989.

[14] R. A. Tapia. On the role of slack variables in quasi-Nweton methods for constrained optimization. In L. C. W. Dixon and G. P. Szegö, editors, Numerical Optimization of dynamic systems, pages 235-246. North-Holland, 1980.

[15] M.J. Todd and Yinyu Ye. A centered projective algorithm for linear programming. Math. of O.R. 15:508-529, 1990.

[16] Yinyu Ye. Interior point algorithms for quadratic programming. Technical report, Dept. of Management Sciences, The University of Iowa, 1989. Working Paper Series No. 89-29.

[17] Yinyu Ye. Interior point algorithms for global optimization. Annals of O.R. 25:59-74, 1990.

[18] Yin Zhang, R. A. Tapia, and J. E. Dennis. On the superlinear and quadratic convergence of primal-dual interior point linear programming algorithms. Technical Report TR90-6, Dept. Mathematical Sciences, Rice University, 1990. To appear in SIAM J. Optimization. 